\title{
Índice de Massa Corpórea, Obesidade Abdominal e Risco de Neoplasia de Cólon: Estudo Prospectivo
}

\author{
Body Mass Index (BMI), Abdominal Obesity and Risk of Colorectal Carcinoma: \\ A Retrospective Study
}

\author{
EDSON JURADODA SILVA ${ }^{1,2}$; ALEXANDRE PELOSI²; ELEODOROCARLOS DEALMEIDA ${ }^{1}$ \\ 1. HSE - RJ (Serviço de Coloproctologia); 2. Casa de Portugal - RJ (Serviço de Endoscopia Digestiva).
}

\begin{abstract}
SILVA EJ; PELOSI A; ALMEIDA EC. Índice de Massa Corpórea, Obesidade Abdominal e Risco de Neoplasia de Cólon: Estudo Prospectivo. Rev bras Coloproct, 2010;30(2): 199-202.

RESUMO: Objetivo: Investigar a associação do excesso de peso e da obesidade abdominal como fator de risco para câncer colorretal/adenoma CCR/Ad. Pacientes e Métodos: De janeiro de 2007 a dezembro de 2008, 1287 pacientes com idade igual ou superior a 50 anos, sem fatores de risco para CCR, foram submetidos a colonoscopia total e avaliados quanto ao índice de massa corpórea IMC e razão cintura quadril RCQ. Teste t de Student foi usado para estudo da média e qui-quadrado para análise de números absolutos. P menor que 0,05 foi considerado significativo. Resultados: Neoplasia foi encontrada em $542(42,1 \%)$, sendo $231(50,7 \%)$ homens e $311(37,3 \%)$ mulheres. IMC $\geq 30$ ocorreu em $43(18,6 \%)$ de CCR/Ad masculino e $84(27 \%)$ feminino P> 0,05. Em 188 (49,4\%) homens com IMC < que 30 ocorreu CCR/Ad, nas mulheres em 227 (36\%) P> 0,05. RCQ foi de $0,96 \pm 0,05 \mathrm{~cm}$ para homens com tumor e $0,94 \pm 0,07 \mathrm{sem}$ tumor $P<0,01$. Nas mulheres RCQ foi de $0,89 \pm 0,06$ com tumor para $\mathbf{0 , 8 8 \pm 0 , 0 6}$ sem tumor $\mathbf{P}<\mathbf{0 , 0 5}$. Conclusões: IMC não está associado a neoplasia de cólon, porem a obesidade abdominal seria fator de risco.
\end{abstract}

Descritores: Câncer de cólon, adenoma de cólon, obesidade, índice de massa corpórea, fatores de risco.

\section{INTRODUÇÃO}

Obesidade está descrita como associada ao aumento no risco do câncer colorretal CCR e do adenoma Ad, sendo a prevalência mais nítida no sexo masculino. ${ }^{1,2,3,4,5}$ Questiona-se no entanto, como fator mais importante, não a obesidade em si, quantificada pelo índice de massa corpórea IMC, mas a presença da obesidade visceral, melhor identificada pela distribuição andróide da gordura, podendo ser indiretamente estudada pela medida da circunferência abdominal ou melhor ainda, pela razão cintura quadril RCQ. ${ }^{6,7,8,9}$, $10,11,12$

O objetivo deste trabalho foi avaliar estas duas variáveis como fator de risco no desenvolvimento do CCR/Ad em nosso meio.

\section{PACIENTES E MÉTODO}

De janeiro a dezembro de 2008 foram realizadas 1287 colonoscopias totais em duas instituições médicas, tendo os pacientes idade mínima de 50 anos e IMC e RCQ calculados. Foram excluídos do estudo, portadores de doença hepática e ou renal crônica, doença inflamatória intestinal, adenomatose familiar FAP, CCR não associado a polipose HNPCC, síndrome de Lynch. Foram considerados obesos os portadores de $\mathrm{IMC} \geq 30 \mathrm{~kg} / \mathrm{m}(2)$. O IMC foi avaliado $\mathrm{em} \mathrm{kg} / \mathrm{m}(2)$ e a RCQ medida em centímetros. O Teste $t$ de Student foi usado para estudo da média e desvio padrão e qui-quadrado para comparar números absolutos. A significância estatística foi considerada quando $\mathrm{P}<0,05$. Todos assinaram consentimento in-

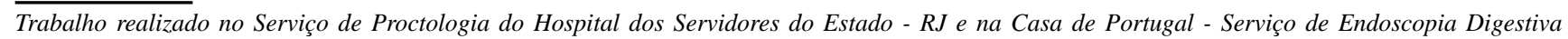
- Rio de Janeiro - RJ - Brasil. 
formado para pesquisa de acordo com o Comitê de Ética Médica Hospitalar.

\section{RESULTADOS}

Houve predominância de procedimentos em mulheres, como podemos ver na Tabela 1.

A faixa etária dos pacientes submetidos ao estudo se encontra na Tabela 2.

O IMC do grupo estudado pode ser visto na curva de Gauss e é mostrado na Tabela 3.

Portadores de neoplasias, CCR/ Ad são mostrados na Tabela 4.

Neste grupo de 542 pacientes, tivemos 250 com CCR (19\%) e 291 (23\%) portadores de adenoma.

Apesar de termos quantidade maior de exames no sexo feminino, foi no sexo masculino onde encontramos a maior incidência de neoplasia, que pode ser visto na Tabela 5.

A distribuição de neoplasia tendo por base IMC, separando obesos de não obesos por sexo podem ser vista na Tabela 6 e tabela 7 a seguir.

A RCQ comparando portadores de neoplasia separados por sexo pode ser vista na Tabela 8 .

\section{DISCUSSÃO}

Apesar da literatura corrente identificar obesidade e afins, tais como síndrome metabólica e obesidade visceral, dentre os fatores de risco para neoplasia de cólon, encontramos divergência ${ }^{13}$ e nossos achados corroboram com isso. Em 1287 pacientes com idade mínima de 50 anos, IMC entre 22,2 e 31,4, média de 26,8 $\pm 4,6$, encontramos 277 indivíduos obesos, $21,5 \%$ da amostra, assim rotulados por terem IMC $\geq 30 \mathrm{~kg} / \mathrm{m}(2)$. Nas Tabelas 6 e 7 observamos que a incidência de neoplasia foi semelhante nos obesos e não obesos, tanto para o sexo masculino como para o sexo feminino.

A incidência de obesidade no Brasil, segundo estimativa do Ministério da Saúde, é de $13 \% .{ }^{14}$ No entanto nos 277 obesos, $21 \%$ em nossa casuística, 127 $(9,8 \%)$ tiveram neoplasia.

Na Tabela 4 vemos que 541 pacientes, $42,1 \%$ da amostra, tiveram neoplasia, constituída por 250 (19\%) com CCR e 291 (23\%) portadores de adenoma. A presença de adenoma nesta faixa etária é semelhante a descrita na literatura ${ }^{15,16,17,18,19}$ e serve também como indicador de qualidade na realização da colonoscopia. ${ }^{20}$
Tabela 1 - Distribuição dos pacientes por sexo.

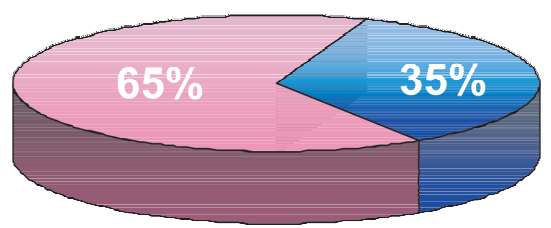

Sexo Masculino - n455

$\square$ Sexo Feminino

- $\mathrm{n}^{\circ} 832$

Total:1287

Tabela 2 - Distribuição por idade em anos.

\begin{tabular}{ll}
\hline Idade em anos & $65,2 \pm 9,8$ \\
Faixa de variação: & $50 \quad-86$ \\
\hline
\end{tabular}

Tabela 3 - IMC do total de pacientes avaliados.

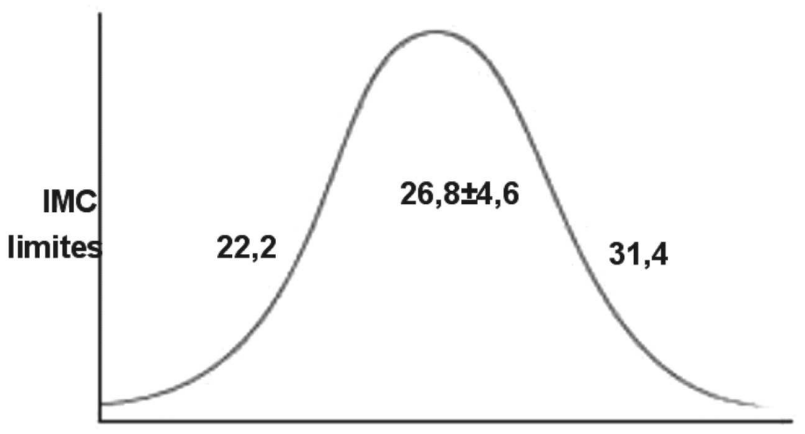

Obesos $N=277(21,5 \%)$

Tabela 4 - Portadores de CCR/Ad.

\begin{tabular}{lll}
\hline & $\mathbf{N}$ & \% \\
\hline CCR/Ad & 542 & 42,1 \\
\hline
\end{tabular}

Tabela 5 - Distribuição de neoplasia CCR/ Ad por sexo.

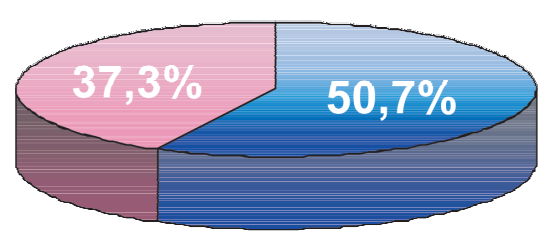

Sexo masculino - n²31

$\square$ Sexo feminino - n०311 
Índice de Massa Corpórea, Obesidade Abdominal e Risco de Neoplasia de Cólon: Estudo Prospectivo

Edson Jurado da Silva e Cols.
Vol. 30
Na Tabela 3 vemos o perfil de nossa casuística, exemplificado na curva de Gauss, onde se percebe que os nossos obesos se encontravam fora da faixa de obesidade mórbida e de super-obesos, caracterizados respectivamente por terem IMC $\geq$ que 40 e $50 \mathrm{~kg} /(\mathrm{m} 2)$. Este fato se explica por não haver nos dois hospitais onde fizemos o estudo setor especializado em cirurgia bariátrica. Temos atualmente protocolo em andamento, para priorizar este grupo de pacientes e com isso obter amostra mais significativa com relação a este tópico.

Vemos na Tabela 8 que a obesidade visceral, identificada pela medida do diâmetro da cintura e ou da razão cintura quadril, atingiu em nosso trabalho, índice de significância para ambos os sexos, mais nítido no masculino e semelhante. ao descrito $\mathrm{em}^{21}$, discor-

Tabela 6 - IMC e CCR/ Ad no sexo masculino.

\begin{tabular}{|c|c|c|c|c|}
\hline & $\mathbf{N}$ & $\%$ & $\mathbf{N}$ & $\%$ \\
\hline & \multicolumn{2}{|c|}{ Com neoplasia } & \multicolumn{2}{|c|}{ Sem neoplasia } \\
\hline $\mathrm{IMC} \geq 30$ & 43 & 16,6 & 32 & 14,2 \\
\hline $\mathrm{IMC}<30$ & 188 & 81,3 & 192 & 85,7 \\
\hline
\end{tabular}

$P>0,05$ dante no entanto $\mathrm{em}^{22}$, pois no primeiro, a obesidade visceral seria o principal fator de risco, no segundo o IMC seria relevante na recorrência de adenoma avançado no homem.

\section{CONCLUSÕES}

Nossos achados sugerem que a obesidade, estimada pelo IMC não estaria associada a maior incidência do CCR/ Ad, porém a obesidade visceral, caracterizada pela $R C Q$, seria fator de risco, mais nítido no homem do que na mulher.

Temos atualmente protocolo de continuidade de estudo, agora trabalhando com obesos mórbidos e super-obesos com a finalidade de ter amostra mais significativa com relação a este tópico.

Tabela 7 - IMC e CCR/ Ad no sexo feminino.

\begin{tabular}{|c|c|c|c|c|}
\hline & $\mathbf{N}$ & $\%$ & $\mathbf{N}$ & $\%$ \\
\hline & \multicolumn{2}{|c|}{ Com neoplasia } & \multicolumn{2}{|c|}{ Sem neoplasia } \\
\hline $\mathrm{MC} \geq 3 \mathrm{C}$ & 84 & 27 & 118 & 22,6 \\
\hline $\mathrm{IMC}<30$ & 227 & 72,9 & 403 & 77,3 \\
\hline
\end{tabular}

$P>0,05$

Tabela 8 - RCQ com relação à presença de neoplasia.

\begin{tabular}{lccc}
\hline & Com neoplasia & Sem neoplasia & P \\
\hline Sexo masculino & $0,96 \pm 0,05$ & $0,94 \pm 0,07$ & $<0,01$ \\
Sexo feminino & $0,89 \pm 0,06$ & $0,88 \pm 0,06$ & $<0,05$ \\
\hline
\end{tabular}

ABSTRACT: Purpose: To investigate the association of overweight and abdominal fat with CRC/Ad. Methods: From January 2007 to December 2008, 1287 patients over 50 years of age, without known risk factors for CRC/Ad (455 men and 832 women), were submitted to a complete colonoscopy and had the BMI and WHR calculated. Student t-test was used for statistical analysis of means and chi-square to compare absolute numbers. A significant $P$-value was defined as $<\mathbf{0 , 0 5}$. Results: Neoplastic lesions were found in $542(42,1 \%)$ subjects, being $231(50,7 \%)$ male and $311(37,3 \%)$ female. BMI $\geq 30$ led to $43(18,6 \%)$ CRC/Ad male and $84(27 \%)$ female $P>0,05$ Patients with $B M I<30$ were found to have CRC/Ad $188(49,4 \%)$ male and $227(36 \%)$ female P> $0,05$. WHR was $0,96 \pm 0,05 \mathrm{~cm}$ for male with tumor and $0,94 \pm 0,07$ without tumor $P<0,01$. For female WHR was $0,89 \pm 0,06$ with tumor and $0,88 \pm 0,06$ without tumor $P<0,05$. Conclusions: These findings suggests that $\mathrm{BMI}$ was not associated with $\mathrm{CRC} / \mathrm{Ad}$ but $\mathrm{WHR}$ could be a predictor for an increased risk of colon neoplasia.

Key word: Colon Cancer; Colon Adenoma; Obesity; BMI; Risk factors. 


\section{REFERÊNCIAS}

1. Bergstrom A, Pisani P, Tenet V, Wolk A, Adami HO. Overweight as avoidable cause of câncer in Europe. Int J Cancer 2001;91(3):421-30.

2. Hou L, Ji BT, Blair A, Dai Q, Gao YT, Potter JD et al. Body mass index and colon cancer risk in Chinese people: menopause as an effect modifier. Eur J Cancer 2006;42(1):84-0

3. Kim SE, Shim KN, Jung SA, Yoo K, Moon IH. An association between obesity and the prevalence of colonic adenoma according to age and gender. J Gastroenterol 2007;42(8):61623.

4. Murphy TK, Calle EE, Rodriguez C, Kahn HS, Thun MJ. Body mass index and colon cancer mortality in a large prospective study. Am J Epidemiol 2000;152(9):847-54.

5. Russo A, Franceschi S, La Vecchia C, Dal Maso L, Montella $\mathrm{M}$, Conti E et al. Body size and colorectal- cancer risk. Int J Cancer 1998;78(2):161-5.

6. Abu-Abid S, Szold A, Klausner J. Obesity and cancer. J Med 2002;33(1-4):73-6.

7. Caan BJ, Coates AO, Slattery ML, Potter JD, Quesenberry $\mathrm{CP}$, Edwards SM. Int $\mathrm{J}$ Obes Relat Metab Disord 1998;22(2):178-4.

8. Kono S, Handa K, Hayabuchi H, Kiyohara C, Inoue H, Marugame $\mathrm{T}$ et al. Obesity, weight gain and risk of colon adenomas in Japanese men. Jpn J Cancer Res 1999;90(8):80511.

9. Moon HG, Ju YT, Jeong CY, Jung EJ, Lee YJ Hong SC et al. Visceral obesity may affect oncologic outcome in patients with colorectal cancer. Ann Surg Oncol 2008;15(7):1918-22.

10. Moore LL, Bradlee ML, Singer MR, Splansky GL, Proctor $\mathrm{MH}$, Ellison RC et al. BMI and waist circumference as predictors of lifetime colon cancer risk in Framingham Study adults. Int J Obes Relat Metab Disord 2004;28(4):559-67.

11. Price GM, Uauy R, Breeze E, Bulpitt CJ, Fletcher AE. Weight, shape, and mortality risk in older persons: elevated waist-hip ratio, not high body mass index, is associated with a greater risk of death. Am J Nutr 2006;84(2):449-60.

12. Wang Y, Jacobs EJ, Patel AV, Rodriguez C, McCullough ML, Thun MJ et al. A prospective study of waist circumference and body mass index in relation to colorectal cancer incidence. Cancer Causes Control 2008;19(7):783-92.
13. Wallace K, Baron JA, Karagas MR, Cole BF, Byers T, Beach MA, et al. The association of physical activity and body mass index with the risk of large bowel polyps. Cancer Epidemiol Biomarkers Prev 2005;14 (9): 2082-6.

14. Reportagens especiais. Portal da Saúde. Ministério da Saúde. www.Saúde.gov.br. Acesso: 08/11/2009

15. Betés M, Muños-Navas MA, Duque JM, Angós R, Macias E, Subtil JC et al. Use of colonoscopy as a primary screening test for colorectal câncer in average risk people. Am J Gastroenterol 2003;98 (12):2648-54.

16. Paspatis GA, Papanikolaou N, Zois E, Michalodimitrakis E. Prevalence of polipys and diverticulosis of the large bowel in the Cretan population. Int J Colorectal Dis 2001;16 (4):25761.

17. Pierzchajlo RP, Ackermann RJ, Vogel RL. Colonoscopy performed by a familiy physichian. A case series of 751 procedures. J Fam Pract 1997;44 (5): 473-80.

18. Rex DK, Mark D, Clarke B, Lappas JC, Lehman GA. Colonoscopy evaluations: justification by cost? Am J Gastroenterol 1996;91 (3):614-5.

19. Rundle AG, Lebwohl B, Vogel R, Levine S, Neugut AL. Colonoscopic screening in average-risk individualas age 40 to 49 vs 50 to 59 years. Gastroenterology 2008;134 (5): 13115.

20. Rex DK. Quality in colonoscopy: cecal intubation first, then what? Am J Gastroenterol 2006;101 (4):732-4.

21. Kim JH, Lim YJ, Kim IH, Sung IK, Shim SG, Oh SO et al. Is metabolic syndrome a risk factor for colorectal adenoma? Cancer Epidemiol Biomarkers Prev 2007;16 (8):1543-6

22. Jacobs ET, Martinez ME, Alberts DS, Jiang R, Lance P, Lowe KA et al. Association between body size and colorectal adenoma recurrence. Clin Gastroenterol hepatol 2007;5 (8):982-90

Endereço para correspondência: EDSON JURADO DA SILVA

Rua Rodolfo de Souza 105 - Vila Isabel

Rio de Janeiro, RJ

20551-270

FAX: (21) 2293551

E-mail: edsonjurado@alternex.com.br 